\title{
Role of iron chelation in hemorrhagic myocardial infarction: a quantitative CMR study
}

\author{
Nilesh R Ghugre ${ }^{1 *}$, Jennifer Barry ${ }^{1}$, John C Wood ${ }^{2}$, Alan Moody ${ }^{3}$, Bradley H Strauss ${ }^{4}$, Graham Wright ${ }^{1,5}$ \\ From 15th Annual SCMR Scientific Sessions \\ Orlando, FL, USA. 2-5 February 2012
}

\begin{abstract}
Summary
Reperfusion hemorrhage is an independent predictor of adverse left-ventricular remodeling following acute myocardial infarction. Iron chelation may potentially alleviate the toxic and pro-inflammatory effects of iron degradation products. CMR can provide insights into the interaction between hemorrhage and iron chelator.
\end{abstract}

\section{Background}

It has been speculated that iron chelation may be beneficial in acute myocardial infarction (AMI) and that early treatment can limit ischemia-reperfusion injury and also reduce infarct size. However, the role of iron chelation in hemorrhagic myocardial infarction, where it would be most suited, has not yet been explored. Reperfusion hemorrhage results in accumulation of iron degradation products of hemoglobin that may be proinflammatory as free iron is toxic in nature. The purpose of the study was to investigate the interaction between iron chelating agent deferiprone (DFP) and hemorrhage in a porcine model of myocardial infarction and monitor remodeling by cardiovascular magnetic resonance (CMR).

\section{Methods}

The study involved two groups of animals that were subjected to a 90 min balloon occlusion of the LAD followed by reperfusion - untreated $(\mathrm{N}=2)$ and DFP treated $(\mathrm{N}=2)$. DFP (supported by ApoPharma Inc., Toronto, $\mathrm{ON}$ ) was administered (orally) a few hours before the procedure (pre-loading) and treatment was continued with a daily dose of $100 \mathrm{mg} / \mathrm{kg}$. Imaging was performed on a 3T MRI scanner (MR 750, GE Healthcare) preAMI (healthy) and day 2-week 4 post-AMI. Edema was evaluated by $\mathrm{T} 2$ quantification using a $\mathrm{T} 2$-prepared

${ }^{1}$ Imaging Research, Sunnybrook Research Institute, Toronto, ON, Canada Full list of author information is available at the end of the article spiral sequence and hemorrhage was identified by $\mathrm{T} 2 *$ determined using a multi-echo gradient-echo acquisition. Infarct assessment was performed by delayed hyperenhancement (DHE) using an IR-GRE sequence.

\section{Results}

Figure 1 demonstrates representative images from the two groups while Figure 2 shows the cumulative time course of the CMR measurements. In the DFP group, hemorrhage was observed only on day 2 and by week 1 it had completely resolved. This was in contrast to the untreated group where resolution of hemorrhage was delayed to week 4 . With DFP, inflammation or edema was substantially reduced by week 4 with T2 values approaching control levels. In the untreated group, edema persisted up to week 4. Ejection fraction (EF) was depressed by week 4 in both groups. However, enddiastolic and end-systolic volumes were relatively unchanged in the DFP group while they increased significantly in the untreated group.

\section{Conclusions}

Hemorrhage may be a source of iron toxicity and a mediator of inflammation, directly contributing to adverse remodeling in the setting of AMI. DFP was able to penetrate the infarct zone and was also effective in neutralizing hemorrhagic byproducts. Elimination of hemorrhage resulted in faster resolution of edema and normal ventricular volumes, representing a beneficial remodeling process. Iron chelation could potentially serve as an adjunctive therapy in hemorrhagic AMI.

\section{Funding}

We would like to acknowledge funding support from the Ontario Research Fund, the Canadian Institutes of Health Research and GE Healthcare. 


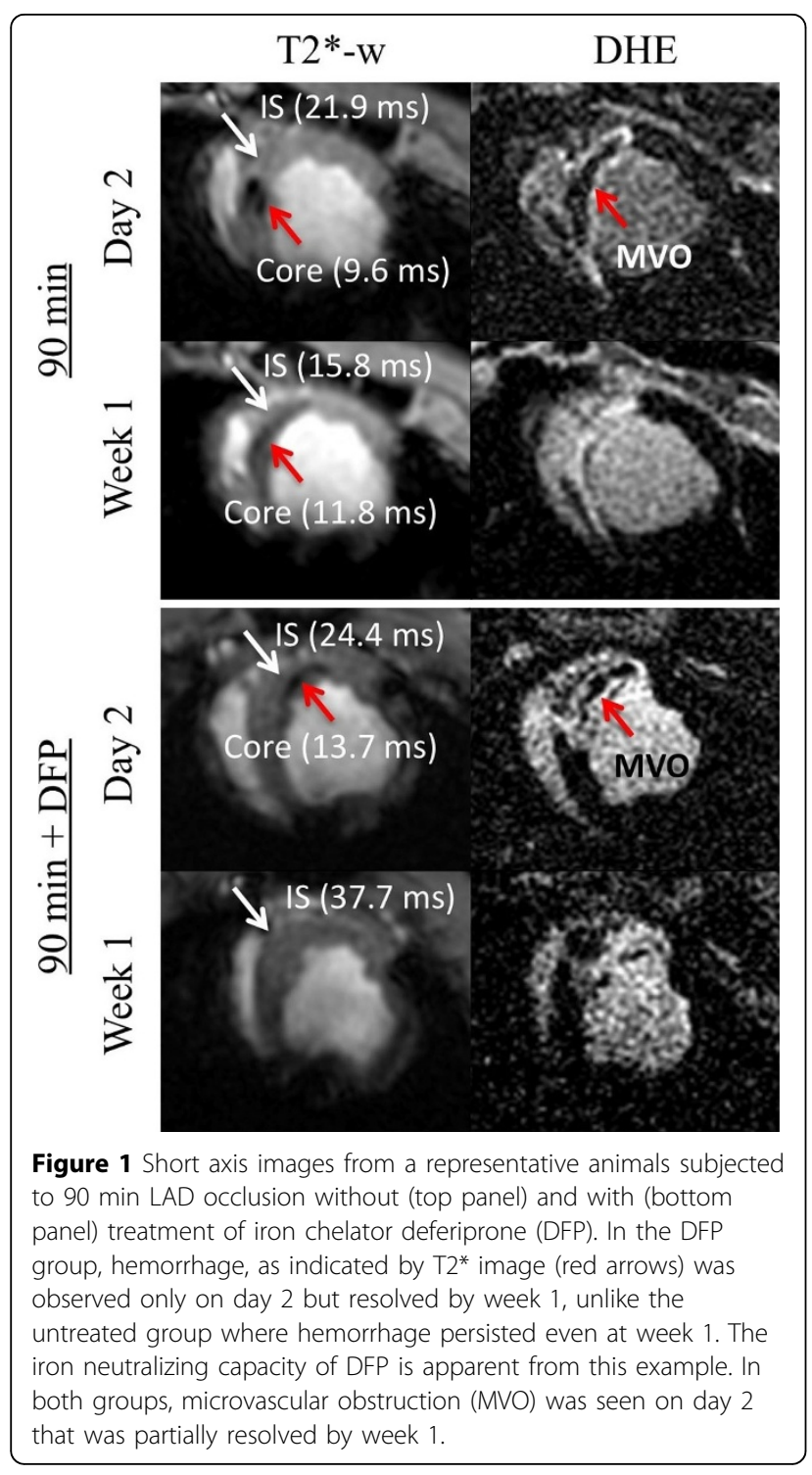

\section{Author details}

${ }^{1}$ Imaging Research, Sunnybrook Research Institute, Toronto, ON, Canada. ${ }^{2}$ Division of Cardiology, Childrens Hospital Los Angeles, Los Angeles, CA, USA. ${ }^{3}$ Department of Medical Imaging, Sunnybrook Health Sciences Centre, Toronto, ON, Canada. ${ }^{4}$ Schulich Heart Program, Sunnybrook Health Sciences Centre, Toronto, ON, Canada. ${ }^{5}$ Department of Medical Biophysics, University of Toronto, Toronto, ON, Canada.

Published: 1 February 2012

doi:10.1186/1532-429X-14-S1-P20

Cite this article as: Ghugre et al:: Role of iron chelation in hemorrhagic myocardial infarction: a quantitative CMR study. Journal of Cardiovascular Magnetic Resonance 2012 14(Suppl 1):P20.
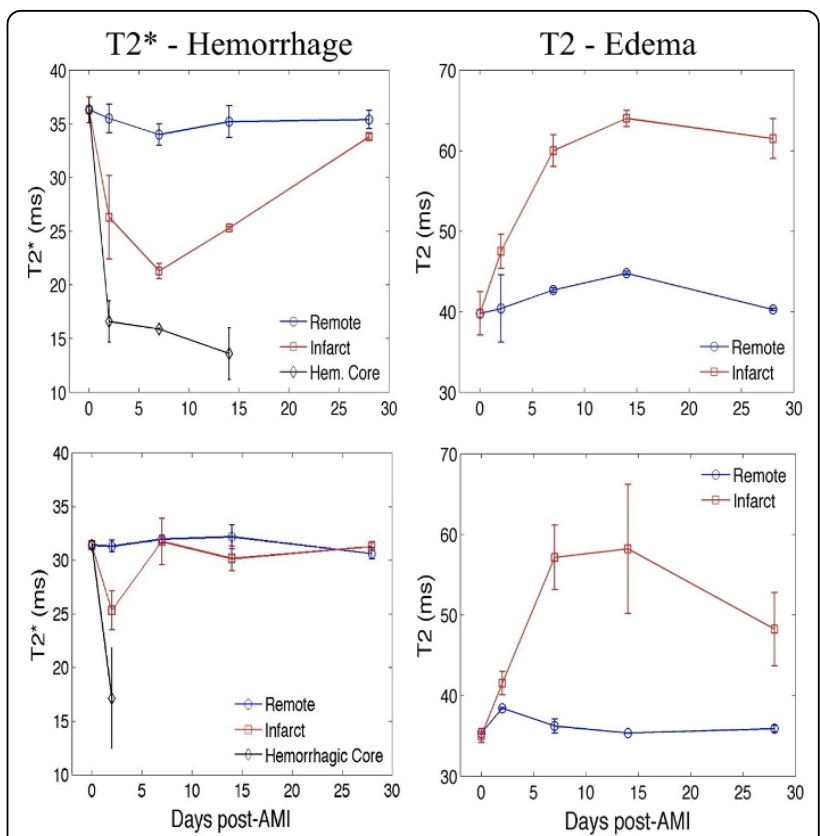

Figure 2 Cumulative time course of $\mathrm{T} 2$ and $\mathrm{T} 2{ }^{*}$ parameters postAMl pooled across all animals $(\mathrm{N}=2)$ in the 90 min infarct untreated (top panel) and treated with deferiprone (DFP, bottom panel); error bars show standard error and day 0 indicates control MRI scans in healthy animals. Infarct zone is shown in red; remote myocardium is shown in blue; hemorrhagic core is shown in black.

\section{Submit your next manuscript to BioMed Central and take full advantage of:}

- Convenient online submission

- Thorough peer review

- No space constraints or color figure charges

- Immediate publication on acceptance

- Inclusion in PubMed, CAS, Scopus and Google Scholar

- Research which is freely available for redistribution 